\title{
A FRAMEWORK FOR AN INFERENTIAL CONCEPTION OF PHYSICAL LAWS
}

\author{
CRISTIAN SOTO \\ Universidad de Chile, CHILE \\ cssotto@uchile.cl \\ OTÁVIO BUENO \\ University of Miami, USA \\ otaviobueno@mac.com
}

\begin{abstract}
We advance a framework for an inferential conception of physical laws, addressing the problem of the application of mathematical structures to the relevant structure of physical domains. Physical laws, we argue, express generalizations that work as rules for deriving physically informative inferences about their target systems, hence guiding us in our interaction with various domains. Our analysis of the application of mathematics to the articulation of physical laws follows a threefold scheme. First, we examine the immersion of the relevant structure of physical domains into mathematical structures. Second, we assess the inferential power of laws resulting from the mathematical formalism employed in the immersion step. And third, we provide a suitable physical interpretation for the extant mathematical structures obtained from the inferential step. We demonstrate that a deflationary, empiricist framework for an inferential conception of physical laws delivers both an understanding of the mathematical character of physical laws, and a way of responding to some of the standard philosophical riddles associated with laws.
\end{abstract}

Keywords: Physical laws $\bullet$ applied mathematics $\bullet$ mapping account $\bullet$ mathematical structures $\bullet$ physical structures $\bullet$ empirical generalizations

\section{Introduction}

The literature on laws of nature has expanded in various directions. The diversity of arguments concerning the character of laws makes it difficult to provide a clear-cut chart to the available positions. A standard map of the debate tells us that three are the main approaches to laws. First, we find a cluster of theories under the label metaphysical views. They share the strategy of extending scientific ontology by metaphysical means, adding layers of universals (Armstrong 1983), dispositions (Bird 2007), or essences (Ellis 2001) to ground laws. Second, a plethora of views are classified as Humean accounts of several sorts, bringing together approaches as disparate as the regularity theory (Carroll 1994), supervenience theories (Loewer 1996; Earman and 
Roberts 2005a, 2005b), and the best system account (Lewis 1986). The latter alone has been further developed in numerous variations, as in the better best system (Cohen and Callender 2009), the perspectivalist better best system (Massimi 2018), and the best system that is best for us (Jaag and Loew 2018). Although with relevant differences, they all develop one form or another of the claim that laws express regularities of some sort. And third, a trend of nomological eliminativism holds the view that not only should we do away with the metaphysicians' laws of nature, but also dispense with the concept of law in our interpretation of scientific practice altogether. A common strategy in this respect consists in replacing the role played by laws with symmetries (van Fraassen 1989) or models (Giere 1988, 1999), both of which, it is argued, can better perform the tasks that were traditionally attributed to laws in representing, explaining or predicting phenomena.

Note that the classification above has its own limits, hence failing to accurately depict the complexities of the debate. We shall concentrate on one such limit, namely: the fact that views above largely overlook the task of making sense of the mathematical character of physical laws, leaving unexplained puzzles arising from the contributions of mathematics to the articulation of laws in scientific practice. ${ }^{1}$ In particular, we examine issues surrounding the application of mathematical structures to the relevant structure of physical domains in the articulation of laws. This, we submit, will help us illuminate at least some of the philosophical riddles associated with laws.

Recent work by Dorato (2005a, 2005b, 2005c) and Woodward (2003, 2013, 2017, 2018) has delivered fruitful, although partial, analyses concerning the mathematical character of physical laws, setting out the scene for us to advance our proposal. $^{2}$ On the one hand, Dorato provides an appraisal of the algorithmic conception that suggests that laws are the software of the universe. Consider the Newtonian inverse-square law of universal gravitation:

$$
F_{\text {grav }}=g m_{1} m_{2} / R^{2}
$$

where $m_{1}$ and $m_{2}$ are the masses of two bodies, $R^{2}$ is the square of the distance between their centers, $g$ is the gravitational constant, and $F_{\text {grav }}$ is the resultant force of gravity. This equation tells us that any two bodies, $m_{1}$ and $m_{2}$, exert a force upon each other that is directly proportional to the product of their masses, varying inversely as the square of the distance between them, $R^{2}$. The law correlates the variables and constant of a mathematical structure with the relevant quantities and structural relations of specific physical systems, such as the Sun-Earth system, and else. At this point, the algorithmic view would argue that mathematics-based law statements can best be conceived as algorithms bridging quantitative data, along with physical constants and the results of the calculus providing explanations and predictions in science. Such algorithms expressing laws would constitute the software of reality, in analogy 
to the computer software operating on a computer hardware (Dorato 2005a, p.61). Laws would enable us to track the probability of one physical system passing from one physical state to another by means of applying the correct algorithm to specific properties of phenomena.

On the other hand, the invariance-based account of laws maintains that "[1]aws are generalizations about repeatable relationships that are invariant over variations in initial and other sorts of conditions, at least within an appropriate range of such variations" (Woodward 2018, p.158). Woodward observes that some philosophers tend to characterize laws in terms of general statements of the form: $(x)(F x \rightarrow G x)$, according to which all $x$ having the property $F$, it has the property $G$. Physical laws, nevertheless, are expressed in terms of differential equations whose peculiarities are many. They may be ordinary, partial, or non-linear differential equations; some of them may have solutions, but others may resist any attempt at finding precise solutions for them; and they are open to the influence of initial conditions (Woodward 2017, pp.12-14). Moreover, differential equations expressing physical laws would yield specific information about their target domain only once additional information about background assumptions has been provided for a specific scenario, and information about supplementary mathematical structure has been added to the original equation in order to index further physical quantities that are relevant to the domain under investigation. Recalling Newton's inverse-square law, the invariance-based account submits that this law remains invariant under a range of interventions: the values of the masses $m_{1}$ and $m_{2}$ continue to be invariant if the distance $R^{2}$ changes over time within certain limits; the known value of the masses can be subject to consideration regardless of background conditions such as the colors of the two bodies or their internal physical constitution. The latter sort of considerations is irrelevant for the determination of the final value of the gravitational force, and it is not represented by additional variables in the formulation of the law statement.

Looking at the intertwining of the philosophy of laws and the philosophy of applied mathematics, in this article we provide an account of mathematics' contributions to the articulation of physical laws. In so doing, we move beyond Dorato's and Woodward's observations. Specifically, we put forward a deflationary, empiricist framework for an inferential conception of physical laws, which casts light on the application of mathematical structures to the relevant structure of physical domains in the articulation of laws in physics. Our use of the inferential conception of the application of mathematics (Bueno and French 2018; Bueno and Colyvan 2011) leads us to label our proposal an inferential conception of physical laws. As will be argued below, laws are empirical hypotheses expressing regularities of various scopes, serving as rules for deriving physically informative inferences about their target domains. The inferential power of physical laws makes them useful tools for guiding our behavior with respect to various issues in scientific practice, such as the construction of 
representations, explanations and predictions of various phenomena. ${ }^{3}$

The structure of our argument is as follows. Section 2 introduces the inferential conception of the application of mathematics, setting the background for us to outline an inferential conception of physical laws in Section 3. Following a threefold scheme, we look into the details of the contribution of mathematics to physical laws in three respects: first, in Section 4 we examine the immersion of the relevant objects and structural relations of physical systems into mathematical structures; then in Section 5 we consider the inferential power that mathematical structures bestow on physical laws; and in Section 6 we assess the challenge of providing a physical interpretation for mathematical structures expressing law statements, especially in view of surplus mathematical structure occurring in laws. After summarizing the main morals from our analysis in Section 7, potential challenges to our framework are considered in Section 8. We close, in Section 9, with a brief concluding remark.

\section{Applying mathematics: an inferential conception}

The general problem of the application of mathematics to the sciences has led to the production of a wide range literature from Wigner's (1960) seminal article, through Steiner's (1998) Pythagoreanism, to a variety of attempts at accounting for the (alleged) unreasonable effectiveness of mathematics in the physical sciences by means of the articulation of some form or another of mathematical platonism (Colyvan 2001; Bangu 2012) or mathematical nominalism (Field 1980 and 1989; Bueno 2016). A broad construal of this general problem considers how mathematics, whose language is abstract and proceeds by means of deductive reasoning, can be so effective in uncovering features of the physical world.

Problems concerning the application of mathematics to physical science have been addressed in terms of elaborations of the mapping account, which has found various formulations in the literature (see Pincock 2004; Bueno and Colyvan 2011; Nguyen and Frigg 2017; and for a critical appraisal, see Rizza 2013). From this viewpoint, mathematical models work like maps by representing features of their target systems and allowing researchers to use them to investigate the physical world. At one extreme, we find advocates of isomorphic mapping relations between high-level mathematical theory and low-level mathematical models, where only the latter are intended to represent the behavior of actual physical systems (van Fraassen 1980, 1989). According to this proposal, a mathematics-based, low-level model can be used to express the empirical adequacy of a high-level mathematized physical theory. At the other extreme, some understand mappings in terms of similarity-based representations (Giere 1988, 1999), arguing that mathematical structures (images, toy models, etc.) work as maps representing relevant structural similarities of their in- 
tended target systems. Whereas the isomorphism-based mapping account establishes a 1-to-1 correlation between a mathematical structure and its target, the similaritybased approach picks out similarities between the relevant structural features of the representation and the relevant structural features of its target physical system.

These forms of the mapping account face several difficulties. ${ }^{4}$ First, neither isomorphism nor similarity deliver a sound criterion for how representation works in physical science. Second, without substantial additional resources, these proposals do not provide an account of how relevant features of the target systems are identified, hence failing to make sense of such criteria as salience and stability of structural features of specific target systems. Third, these proposals, at least as developed so far by their advocates, do not account for the pragmatic character of the selection process of one mathematical structure over another, nor do they accommodate the process of construction of new mathematical structures in applied mathematics. And fourth, they currently do not explain the role played by surplus mathematical structure in scientific reasoning.

A recent development in the debate consists in the elaboration of the inferential conception of the application of mathematics to physical sciences. The inferential conception addresses the specificities of the application of mathematical structures to the relevant structure of physical systems advancing a threefold scheme:

i. Immersion: the first step consists in "establishing a mapping from the physical (empirical/theoretical) situation to a convenient mathematical structure";

ii. Inference: the second step consists in "drawing consequences from the mathematical formalism, using the mathematical structure obtained in the immersion step";

iii. Interpretation: finally, one interprets "the mathematical consequences that were obtained in the derivation step in terms of the initial theoretical set-up" (Bueno and French 2018, pp.52-3).

This framework highlights the main dimensions of the application of mathematics, from establishing the relevant mapping, through the derivation of appropriate inferences from the chosen mathematical structure, to providing a physical interpretation for the resultant mathematical structure, hence returning to the initial problem situation. Among its key aspects, we will consider the following three in our elaboration of an inferential conception of physical laws. First, the inferential conception of the application of mathematics fully embraces pragmatic criteria involved in the process of applying mathematics. For establishing the initial mapping, a convenient mathematical structure is chosen. If two or more mathematical structures are available for addressing a domain, a selection is made based on pragmatic criteria, such as simplicity, tractability, and so on. In some cases, if no mathematics is available for 
suitably mapping a given target domain, a specific mathematical formalism can be built up. Likewise, concerning the target domain, when drawing mappings, scientists identify the relevant quantities and relations thereof whose salience and stability inform us about the structure that is interesting from the viewpoint of the problem situation. We do not expect mathematical mappings to cover thoroughly a domain, but only to selectively represent those features that are relevant for the problem at hand. The goal in the end is this: at least some of the consequences derived from the mathematics employed in mappings are expected to be physically informative about the relevant target domain.

A second element of the inferential conception of the application of mathematics that will become decisive for our account of physical laws has to do with the adoption of the partial structures approach (da Costa and French 2000, 2003), which provides a framework for understanding how scientific representation works. From this perspective, the mapping from a physical domain to a suitable mathematical structure can be introduced as follows: ${ }^{5}$

- Be $W$ the relevant structure of a physical domain which we seek to map;

- Be $M$ the mathematical structure which we implement to map $W$; and,

- Be $\Phi$ the mapping relation $W \rightarrow M$.

Various considerations are in order. First, the mapping $\Phi(W \rightarrow M)$ represents a kind of morphism which, according to the inferential conception, can take the form of a partial isomorphism or partial homomorphism. Second, assuming a partial morphism, $M$ may contain more structure than $W$, or vice versa. And third, the information yielded by $\Phi(W \rightarrow M)$ has an open structure, accommodating elements of $M$, which at a certain point we ignore if they have a counterpart in $W$.

Set theoretically, we express the relation of partial isomorphism or partial homomorphism as follows. A partial structure is an ordered tuple $\left\langle D, R_{i}\right\rangle_{i \in I}$, where $D$ is a non-empty set and $R_{i}, i \in I$, is a family of partial relations. In turn, each partial relation $R$ can be viewed as an ordered triplet $\left(R_{1}, R_{2}, R_{3}\right)$, where $R_{1}, R_{2}$ and $R_{3}$ are disjoint sets, with $R_{1} \cup R_{2} \cup R_{3}=D^{n}$; and such that

- $R_{1}$ is the set of n-tuples which we know that belong to $R$;

- $R_{2}$ is the set of n-tuples which we know that do not belong to $R$; and

- $R_{3}$ is the set of n-tuples for which we ignore whether or not they belong to $R$.

Hence, by endorsing the partial structures approach, the inferential conception has the means to make sense of the fact that mapping processes selectively pick out relevant (i.e., salient and stable) features of physical systems from the perspective of a problem situation, without purporting to implement a full mapping. Moreover, the theories and hypotheses involved in the application process need not be true, but 
be partially true only, that is, true only relative to a restricted portion of the domain under consideration, and consistent with accepted claims about the domain of application (see da Costa and French 2003).

Yet, a third feature of the inferential conception of the application of mathematics, which will play a role in our understanding of physical laws, is this: granting the adoption of the partial structures approach, the inferential conception has the tools to account for the appearance of surplus mathematical structures in scientific theorizing (Redhead 1975). Physical laws routinely involve the appearance of surplus mathematical structure, which can adequately be identified as $R_{3}$ above. At some point, we ignore whether such surplus mathematical structure belong to the set of relations, $R$, hence belonging to the mapping $\Phi(W \rightarrow M)$. The inferential conception clarifies the roles of surplus mathematical structure, according to which, even though surplus structure may resist physical interpretation, it can still play various roles in scientific theorizing in general and in the articulation of physical laws specifically.

With these considerations in place, we can now elaborate an inferential conception of physical laws, returning to the points above as needed.

\section{Outlining an inferential conception of physical laws}

We outline a framework that accounts for the articulation of laws, particularly in the context of the application of mathematical structures to physical domains. The account shall cast light on both mathematics' applicability and the character of mathematics-based law statements. Here is the threefold scheme for an inferential conception of physical laws:

i. Immersion in laws: One dimension of the contribution of mathematics to the articulation of physical laws consists in establishing a mapping from the relevant structure of a physical system (taking into account its objects, properties and relations) to a suitable mathematical structure.

ii. Inferring form laws: A second dimension of mathematics' contribution to physical laws can be identified with the set of inferences that we can derive from the mathematical formalism employed in the articulation of a law statement. Along with its representational and expressive capacities, mathematics bestows inferential power on laws, facilitating the tasks of explaining and predicting relevant phenomena. Note, at this point, that whether the inferences derived are physically informative is yet to be decided in the third step.

iii. Interpreting laws: A third dimension of the contribution of mathematics to laws consists in providing us with a mathematical space of possibilities that require to pass the test of physical interpretation. That is, we need to work out 
a physical interpretation for the extant mathematical structures resulting from the inferences derived from the initial mathematical formalism (along with any other added mathematical structure). In view of this, two separate tasks are to be undertaken: First, a distinction should be made between physically informative and physically uninformative mathematical inferences, where only the former succeeds in receiving an adequate physical interpretation, thereby yielding relevant information regarding aspects of the phenomena under study. And second, attention is to be paid to cases in which surplus mathematical structure occurs in laws, since even though some structures may lack physical interpretation, they can still play a role in the process of successfully applying mathematics to mathematics-based law statements.

How do we understand physical laws within this framework? We take this framework to be suitable for a deflationary, empiricist account of laws. If a definition of physical laws is required, here is one that can neatly be accommodated by the inferential account: physical laws are empirical hypothesis that serve as rules or directions for deriving physically informative inferences (sometimes along with physically uninformative ones) about their target domains. The inferential power of physical laws, which is strengthened by the mathematical formalism employed in each case, makes them useful tools for guiding our behavior with respect to various physical domains, hence aiding the construction of representations, explanations, and predictions of several phenomena, among the tasks routinely associated with laws. In the next three sections, we examine local scenarios of the application of mathematical structures to the articulation of laws keeping in mind the suggested threefold framework. We consider each step in turn.

\section{Immersion in laws}

The first step of the inferential conception of physical laws consists in the immersion of the relevant structure of a physical domain into the chosen mathematical structure. The selection of the relevant mathematics is inherently pragmatic, including contextual considerations about details imposed by both the target system and the availability of mathematics at a given time.

When it comes to physical laws, mathematical structures are applied to relevant aspects of a broad range of empirical/theoretical situations. Following a familiar distinction, for clarificatory purposes we distinguish between the application of mathematics to phenomenological laws and to fundamental laws. Phenomenological law statements are routinely obtained from directly probing an empirical situation. We have in such cases robust epistemic access to the situation of interest. By contrast, fundamental laws are not obtained in this way, and we usually lack straightforward 
access to their targets. They require the introduction of idealizations and surplus mathematical structure to provide a generalization about their domain. Importantly, it should be noted that our inferential conception of physical laws does not depend on there being a sharp distinction between phenomenological and fundamental laws. Indeed, we are not committed to the claim that physical laws can be accurately classified in terms of exactly these two types of laws. Rather, the lack of sharp boundaries between them is more likely to be found in scientific practice. For the time being, though, we introduce this nomenclature only to highlight features of the application of mathematics to physical laws that should be examined.

In cases of phenomenological laws, their mathematical structure straightforwardly picks out the relevant aspects of physical systems. Consider Hooke's law:

$$
F_{s}=k x
$$

$F_{s}$ is the force needed to compress or extend a spring; $k$ is a constant factor that is assigned to specific sorts of springs in each application of the formalism to a local physical scenario, which ultimately introduces the stiffness of a spring; and $x$ represents the displacement or change in length of a spring. The equation, in brief, represents the relevant structure of a physical system, i.e., a spring that is subject to pressuring or expanding forces, hence expressing a physical law that states an empirical generalization that captures the relevant structural relations embedded in its target system. For some phenomenological laws, the target system may bear further physical elements amenable to be identified be means of additional mathematical variables, as needed. In any case, the mathematical structure employed offers a simplification of the physical situation, indexing only those properties and structural relations that are relevant to the specific scenario. Naturally, other details of physical systems go beyond what can or need to be captured by the mathematical structure expressing a law statement.

More complex, philosophically intriguing scenarios are those of fundamental laws that only indirectly refer to physical systems through the introduction of a variety of mathematical idealizations and surplus mathematical structure. Consider the general form of the time-dependent Schrödinger equation:

$$
i \hbar \frac{d}{d t}|\Psi(t)\rangle=\hat{H}|\Psi(t)\rangle
$$

where $i$ is an imaginary number, $\hbar$ is the reduced Plank constant, and $\Psi$ is the state vector of a quantum system. A series of difficulties are added in this case. This law introduces an imaginary number, the reduced Plank constant, and the state vector of a quantum system, all of which present various difficulties when it comes to performing a mapping from the target physical domain to the mathematical. Imaginary 
numbers, it may be argued, find no counterpart in the world. The equation, indeed, bears more mathematical structure than the physical structure that we can attribute to a target system. Be that as it may, the Schrödinger equation provides information about the wave function of a quantum system evolving in time. To perform applications of this equation, we need to specify the Hamiltonian for the quantum system, considering the kinetic and potential energy of the particles constituting the system in question. Furthermore, as such, it would be inadequate to require for the Schrödinger equation a 1-to-1 isomorphic conservation of the structural relations exhibited in its target system. This equation delivers a space of physical possibilities for a quantum system, which can be adjusted to specific physical scenarios by considering the relevant Hamiltonians in each case. We shall return below to the partial character of mappings performed by such mathematics-based law statements.

In brief, physical laws, whether they are phenomenological or fundamental, are only intended to selectively pick out the relevant structural relations of target systems. It is usually the case that certain physical aspects of the relevant target escape the domain of interest of mathematical structures expressing laws. And the process of applying mathematics to the articulation of physical laws, we noted, is pragmatic in character. Depending on the situation under consideration, we may find that, given the relevant properties and relations of a target system, it may be the case that one piece of mathematical structure be more suitable to successfully perform the mapping than other pieces of mathematics available at a time. Yet, we may lack a suitable mathematical structure to map the relevant quantities and relations embodied in the target at hand, as in the well-documented case of Newton's introduction of infinitesimal calculus in classical mechanics. In a different scenario, scientists may have at their disposal two or more mathematical structures that can be used in the partial mappings of the phenomena under investigation, the problem being having to find a criterion (or set of criteria) for choosing one among them. ${ }^{6}$

\section{The inferential character of laws}

Recall that the second step of the inferential conception of the application of mathematics consists in "drawing consequences from the mathematical formalism, using the mathematical structure obtained in the immersion step" (Bueno and French 2018, p.52). This is "the key point of the application process, where consequences from the mathematical formalism are generated" (Bueno and French 2018, p.53). We shall show now that the same goes for the inferential conception of physical laws.

Physical laws perform various tasks in scientific practice. It has been widely acknowledged that law statements facilitate the axiomatic presentation of theories in their domain, occupying a high-level in the structure of scientific theorizing in their 
fields. Likewise, physical laws enhance theoretical and physical unification in the sciences, strengthening intra- and inter-theoretical relations. Cases of this sort abound in the history of physics, standard examples being the unification of Kepler's laws of celestial mechanics and Galileo's laws of terrestrial motion by means of Newton's second dynamical law and the law of universal gravitation; or the Maxwellian unification of physical optics, electric and magnetic theories.

What interests us at present is to highlight the inferential power of physical laws. Mathematics-based law statements work as rules for deriving physically informative inferences about their target systems. Consider, for instance, the differential equation that Paul Dirac identified (now called Dirac's equation), which contains negative energy solutions (for references and details, see Bueno 2005). The fact that the equation has such negative solutions is an inferential aspect of the mathematics used in the formulation of the equation. By embedding the relevant physical situation, involving energy, momentum and mass, into the suitable mathematical model, the negative energy solutions result. The crucial issue is then to articulate a physically relevant interpretation of such solutions, and it was precisely by identifying such an interpretation (after two false starts) that Dirac was eventually able to posit the positron as a suitable physical interpretation of the negative energy solutions. This takes us to the next step of our approach.

\section{Interpreting physical laws}

The third step of the inferential conception of the application of mathematics suggests interpreting "the mathematical consequences that were obtained in the derivation step in terms of the initial empirical set up" (Bueno and French 2018, p.53), thereby establishing a mapping from the resultant mathematical structure to the initial theoretical/empirical situation. When it comes to interpreting mathematics-based law statements, such interpretation takes place at two levels. On the one hand, a partial mapping is established between the mathematical structure that is chosen to formulate a physical law and the relevant structure of its target domain, as was noted in the immersion step in Section 4 above. And on the other, once inferences allowed by the mathematical formalism have been derived in the inferential step (Section 5 above), additional partial mappings are to be introduced in order to provide a suitable physical interpretation for the extant mathematical structure.

The scope of possible physical interpretations of a mathematically expressed physical law is ultimately determined by its target domain. Once more, cases of phenomenological laws routinely allow inferences whose interpretation may usually prove to be straightforward. As in the case of the abovementioned Hooke's law, in appropriate contexts, we have robust epistemic access to linear springs, hence deter- 
mining the values for $F_{s}, k$, and $x$. Moreover, applications of Hooke's law to continuous media and to analogous models of the harmonic oscillator can be developed, in each such scenario being possible to determine the physical quantities that fill in the variables and relevant constant.

However, when it comes to providing interpretations of explanations or predictions derived from fundamental laws, things get more complicated. Cartwright (1999) has famously argued that fundamental laws are simply false when taken literally, failing to apply directly to the world. Our framework for an inferential conception of physical laws has the means to accommodate this concern. In fact, the immersioninference-interpretation scheme takes into consideration the relevant details involved in connecting mathematical and physical structures. Consider Cartwright's example of Newton's second dynamical law:

$$
F=m a
$$

To get from the mathematics to their intended target physical domain, we firstly need to recognize that the equation in question already bears physical interpretation, i.e., it refers to a certain force, $F$; to the mass of a body, $m$; and to its respective acceleration, $a$. As an inferential device, this equation enables us to construct various models that help us bridge the representational gap between a mathematical structure and the relevant structure of the target physical domain. These models will, in turn, add further mathematical structures to the initial mathematics-based law statement, including variables indexing additional features of the intended target system (such as air resistance) that allow one to achieve a more accurate mapping of the relevant structural relations.

Here is an example: for a body of mass $m$ that is affected by forces $F_{1}, F_{2}, F_{3}$ and $F_{4}$, we have the following diagrammatic presentation that considers the direction of the forces:

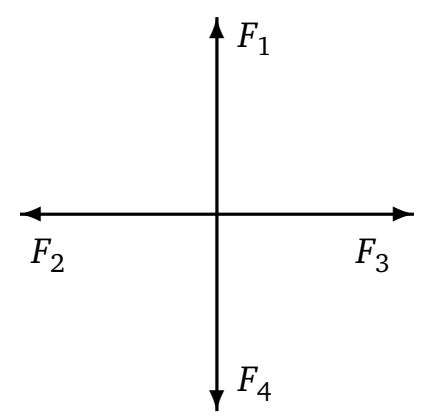

Forces $F_{2}$ and $F_{3}$ affect the horizontal direction. Applying the second law, we obtain:

$$
a_{x}=\sum F_{x} / m=F_{2}+F_{3} / m
$$


And the same goes for the acceleration along the vertical direction for $F_{1}$ and $F_{4}$ :

$$
a_{y}=\sum F_{y} / m=F_{1}+F_{4} / m
$$

Moreover, the inferential power of the second dynamical law can be employed to solve physical problems associated with Hooke's law mentioned earlier, moving now from the general form $F=m a$ to

$$
F=m a=m \cdot d^{2} x / d t^{2}=-k x
$$

where $d^{2} x / d t^{2}$ corresponds to the second derivative of the mass of the body with respect to time and $k$ is the constant of proportionality introduced in Hooke's law, this time applied to a model of a linear oscillator. ${ }^{7}$

In brief, we grant that mathematical statements expressing physical laws can be given a physical interpretation (as in the cases of the Hooke law, the Schrödinger equation, or the Newtonian second dynamical law), so long as it is possible to determine their own physical scope, which is given by the physical generalization they express about a target domain. Regardless of whether they are fundamental or phenomenological, physical laws are intended to refer to their instances. The task we need to perform is to clarify the initial partial mapping relation (immersion step), to derive physically informative inferences from the mathematical structures expressing the law, and to interpret the extant mathematical structures obtained in terms of the initial empirical set up. Some inferences may be completed in a few steps, as in the case of Hooke's law, whereas other may require longer, more complex inferential chains before latching onto the relevant structure of a target physical domain.

\section{Morals from the inferential conception of physical laws}

Thus far, we have outlined a framework for an inferential conception of physical laws, focusing on various dimensions of the application of mathematical structures to the relevant structure of physical domains in the articulation of laws. We are aware that our proposal is broadly programmatic. But we believe it effectively captures some important features of mathematics' contributions to the articulation of laws. In what follows, we highlight some of the benefits of our framework.

The first benefit consists in addressing philosophical riddles that arise from the application of mathematics to the articulation of physical laws. Standard formulations of metaphysical views, Humean accounts, and eliminativist approaches have largely overlooked the roles of mathematics in physical laws (see Section 1 above). As we mentioned earlier, Dorato (2005a, 2005b, 2005c) and Woodward (2017, 2018) constitute exceptions to this trend: the former sets forth an appraisal of the algorithmic view of laws, whereas the latter examines the fact that most physical laws 
are expressed in terms of differential equations. Our framework moves beyond their contributions by providing a setting in which a variety of connections between mathematical and physical structures can be represented in the context of the formulation and exploration of physical laws. As noted, three dimensions of the application of mathematics to physical laws are distinguished, namely: the immersion step, in which a map from the relevant structure of a target domain to a suitable mathematical structure is specified; the inferential step, in which consequences from the mathematical formalism are derived; and the interpretation step, in which a physical interpretation is provided for the extant mathematical structure.

A second benefit of our framework consists in setting the groundwork for the development of a philosophical conception of physical laws. The inferential conception embraces the fact that most physical laws are mathematics-based statements. Scientists construct laws and use them as guides about regularities in specific domains. Such mathematics-based physical laws are not to be assessed in terms of philosophical criteria concerning the universality, necessity, truth and objectivity of the metaphysicians' laws of nature, since it is not clear that such criteria can (or need to) be met (see van Fraassen 1989). By contrast, our framework acknowledges that physical laws are contingent, delivering partial truths about their domains. They may turn out to be false because experiments may reveal unforeseen features of a physical domain, or because the mathematical framework employed in their expression can be revised and corrected. Despite that, as they stand, physical laws provide empirical hypotheses that express generalizations about the behavior of phenomena in their domains.

Where does the inferential conception of laws of nature stand on the debate concerning the character of laws? We can only make some brief remarks at this point. Let us consider the traditional distinction between metaphysical, Humean, and eliminativist accounts of laws:

- Against metaphysical views,

- we endorse an empiricist epistemology and its concomitant ontology in our analysis of physical laws, thereby rejecting the postulation of special entities to ground the nomic status of law statements.

- Against Humean views,

- we have no quarrel with endorsing the modalities of physical domains, and we see no need to reduce laws to a non-modal Humean basis.

- Against nomological eliminativism,

- we suggest an account of the physical laws that we find in scientific practice, hence dispensing with revisionary interpretations of science.

Of course, much more needs to be said about these issues, and we will do so in future 
work. However, for the time being, let this suffice to place the inferential conception of physical laws in the debate.

A third benefit of an inferential conception of physical laws consists in putting forward an account of the scope of physical laws that remains close to what we find in physical science. The adoption of the partial structures approach (Section 2 above) contributes to this goal. Mathematically expressed physical laws abstract away several aspects of physical domains, introducing idealizations about unrealized (and sometimes in principle unrealizable) physical situations (as in the case of infinite idealizations). The partial structures approach that underpins our defense of the inferential conception of physical laws acknowledges the complexities involved in interpreting physical laws, making sense of the fact that standard textbook formulations of fundamental laws (say, $F=m a$ ) should be interpreted as empirical hypotheses providing a generalization about a physical domain. Such laws are meant to serve as rules for deriving inferences and building models of concrete situations once a suitable additional mathematical machinery (additional variables, functions, and else) has been added to index new physical information. What matters about fundamental laws is that they provide a mathematical space for the realization of physical possibilities in a domain. To interpret them as literal, first-hand descriptions of specific physical situations (as Cartwright 1999 suggests) would be to overlook their roles in the sciences' inferential practices. The partial structures approach has the flexibility to accommodate partial mapping relations in cases in which the relevant physical domain may have more structure than the structure we need to introduce in the articulation of the respective mathematics-based law statement, as well as cases in which mathematics-based law statements have more structure than the structure that is available in the corresponding physical domain.

This consideration leads us to a fourth benefit of our framework: it can account for the occurrence of surplus mathematical structure in laws. Even though the formulation of some laws requires the introduction of surplus mathematical structure, it can still be shown that, on the one hand, such laws are physically informative about relevant phenomena; whereas on the other, the surplus mathematical content plays heuristic roles, facilitating the solutions of equations and enabling us to derive physically informative inferences about the relevant domain in each case. The occurrence of surplus mathematical structure need not entail the view that laws are purely mathematical devices, nor the idea that the world is mathematical in character. More will be said about these benefits in future work. 


\section{Counterarguments to our framework, and their responses}

We have outlined a framework for physical laws that takes into account the application of mathematics. We explore now the way in which our proposal responds to additional philosophical concerns. One is this. We speak of applying mathematical structures to the relevant structure of physical domains. We have been explicit about the pragmatic character of this task, both at the level of selection of a convenient mathematical structure and at the level of the identification of the relevant structures of a physical domain. In so doing, a potential challenge to our view would question whether we can offer a criterion (or set of criteria) for distinguishing between mathematical and physical structures. This challenge gains momentum once we consider recent trends in the philosophy of science, particularly in the philosophy of physics and mathematics, where such a distinction has come to be questioned (Tegmark 2014; Ladyman and Ross 2007). Although we cannot fully address this concern in this article, it should be noted that our empiricist framework for an inferential conception of physical laws need not endorse ontological commitment to mathematical and physical structures. It suffices for our purposes to maintain that mathematical structures are abstract, whereas physical structures are concrete. Structural relations among physical quantities are partially described by suitably interpreted mathematical structures. Scientific research typically detects structural relations among physical properties or quantities, refines one's access to them, and track them spatiotemporally (Bueno 2019). In the case of laws, this is achieved by exploring their inferential resources allowed for by suitably interpreted mathematical structures.

A second challenge builds up from the first: it can be argued that we do not tackle the issue of whether the world comes structured or unstructured (for instance, whether it is orderly or disorderly, deterministic or indeterministic) as part of the account we favour of the application of mathematics to the articulation of physical laws. In response, it should be noted that our empiricist framework remains neutral about metaphysical presuppositions concerning the structured or unstructured divide regarding the world. Whether the world is ultimately (un)structured is a metaphysical concern that can be run largely independently from the examination of mathematics' applications to physical laws (and to physical science overall). Moreover, considerations regarding applications of mathematics to physical domains alone would not suffice to settle such a metaphysical concern.

A third objection against the inferential framework concerns the source of modality for physical laws. This concern raises a significant issue, as an account of the source of modality for the nomological status of laws needs to be offered. At present, it should be clear that our account need not presuppose that the modality grounding the nomological character of physical laws is mathematical, and thus provides no constraints on the physical world (except, perhaps, for cardinality considerations). 
Mathematical modality, we take it, involves modal properties of mathematical objects without, however, the commitment to a metaphysical understanding of these properties. For instance, assuming classical logic, it is impossible that there is the greatest natural number, given that positing such an object is inconsistent with the infinitude of such numbers. This is a modal property of natural numbers, whether such numbers exist or not. But what matters with regard to physical laws are modal properties of objects in the physical world rather than features of abstract, causally inert entities.

In making sense of physical modality, we also emphasize the role played by the relevant properties (again, understood in non-metaphysical terms). If a table is breakable it has a modal property: it can break under certain circumstances. This is a feature of the table that results from the materials that constitute it, and it is not clarified by introducing metaphysical entities, such as universals, dispositions, essences, possible worlds, and the like. Our account does not posit such entities at all. In fact, making sense of physical modality in metaphysical terms is a distraction that deviates one from the real issue, which is to understand the physical possibilities or impossibilities in question, rather than some artificially constructed philosophical regimentation. (We will return to these issues in future work; meanwhile, see Bueno and Shalkowski 2015 for additional discussion.)

Our approach should not be in tension with accounts of physical modality that do not invoke extraneous metaphysics. The empiricist framework for laws advanced by the inferential conception seems compatible with a conception of physical modality that rests on the invariance of physical domains (Woodward 2018). The invariance and stability of properties and relations of physical domains are particular features of such domains, which are not specified in terms of metaphysically contentious entities. Of course, far more needs to be said about these issues and we will develop further the details of the empiricist framework of our approach in due course.

A fourth and final objection raises the concern that our inferential conception of physical laws proposes a return to logical positivism and the Vienna Circle, having precedents in Neurath and Schlick, or so it could be argued. ${ }^{8}$ Neurath, for instance, notes: "With the help of observation statements we formulate laws; according to Schlick, these laws are not to be seen as proper statements but as directives for finding predictions of individual courses of events" (Neurath 1983 [1931], p.53, our italics). Similarities between Neurath's interpretation of Schlick and the inferential conception are apparent if we consider that laws are directives for finding predictions. We have indeed claimed that physical laws are empirical hypotheses that work as rules for deriving physically relevant inferences about their target systems. Nevertheless, differences between Neurath's take on laws and our account are substantial. First, we do not adopt an instrumentalist attitude toward laws, according to which such statements lack truth value. In contrast, laws do have a truth value, and are typically 
at best partially true; that is, they are compatible with accepted information about the domain they are about, without fully describing every aspect of such a domain (see da Costa and French 2003). Moreover, with the use of the partial structures framework, the semantic view of theories is favored rather than the syntactic view. Second, we do not restrict the roles of laws to that of finding predictions; laws enable us to articulate explanations and unification in science, and they are used in the construction of models, computer simulations, and else, and all of these features can be understood within an empiricist framework. Finally, nowhere on our account do we invoke observation statements or protocol statements, which were central to logical positivism, with the myriad of problems they yield.

\section{Conclusion}

We have outlined a framework for an inferential conception of physical laws, which provides both an understanding of mathematics' contribution to the articulation of laws and a philosophical conception of the laws that we find in scientific practice, particularly those laws in physics that are routinely expressed in mathematical terms. To do so, we have accordingly moved beyond standard metaphysical, Humean, and eliminativist views on laws. We are more sympathetic to Dorato's examination of mathematics' effectiveness in laws and Woodward's invariance-based conception, albeit there are important differences between the inferential conception and their views.

Rather than engaging in critical discussions, we have chosen to provide a threefold scheme for making sense of the dimensions of the application of mathematical structures to physical domains in the articulation of laws, namely, the immersioninference-interpretation framework. With this, we have aimed to advance a novel perspective to both address issues surrounding the application of mathematics to laws, and outline (only schematically) a response to some concerns in the philosophy of laws of nature related to the structure of the world and modality. Our framework for an inferential conception of physical laws will be developed in these and other directions in future work.

\section{References}

Armstrong, D. 1983. What is a Law of Nature? Cambridge: Cambridge University Press.

Bangu, S. 2012. The Applicability of Mathematics in Science: Indispensability and Ontology. London: Palgrave Macmillan.

Bird, A. 2007. Nature's Metaphysics. Laws ad Properties. Oxford: Oxford University Press. 
Bueno, O. 2005. Dirac and the Dispensability of Mathematics. Studies in History and Philosophy of Modern Physics 36: 465-90.

Bueno, O. 2016. An Anti-Realist Account of the Application of Mathematics. Philosophical Studies 173: 2591-604.

Bueno, O. 2019. Structural Realism, Mathematics, and Ontology. Studies in History and Philosophy of Science 74: 4-9. DOI: https://doi.org/10.1016/j.shpsa.2018.12.005.

Bueno, O.; Colyvan, M. 2011. An Inferential Conception of the Application of Mathematics. Nous 45(2): 345-74.

Bueno, O.; French, S. 2018. Applying Mathematics. Immersion, Inference, Interpretation. Oxford: Oxford University Press.

Bueno, O.; Shalkowski, S. 2015. Modalism and Theoretical Virtues: Toward an Epistemology of Modality. Philosophical Studies 172: 671-89.

Carroll, J. 1994. Laws of Nature. Cambridge, Cambridge University Press.

Cartwright, N. 1999. The Dappled World. A Study of the Boundaries of Science. Cambridge: Cambridge University Press.

Cohen, J.; Callender, C. 2009. A Better Best System Account of Lawhood. Philosophical Studies 145(1): 1-34.

Colyvan, M. 2001. The Indispensability of Mathematics. Oxford: Oxford University Press.

da Costa, N. C. A.; French, S. 2000. Models, Theories, and Structures: Thirty Years On. Philosophy of Science 67: S116-S127.

da Costa, N. C. A.; French, S. 2003. Science and Partial Truth: A Unitary Approach to Models and Scientific Reasoning. Oxford: Oxford University Press.

Dieks, D. 2005. The Flexibility of Mathematics. In: G. Biniolo; P. Budinich; M. Trobok (eds.) The Role of Mathematics in Physical Sciences: Interdisciplinary and Physical Aspects, pp.11529. Dordrecht: Springer.

Dorato, M. 2005a. The Software of the Universe. An introduction to the History and Philosophy of Laws of Nature. Aldershot: Ashgate.

Dorato, M. 2005b. The Laws of nature and the Effectiveness of Mathematics. In: G. Biniolo; P. Budinich; M. Trobok (eds.) The Role of Mathematics in Physical Sciences: Interdisciplinary and Physical Aspects, pp.131-44. Dordrecht: Springer.

Dorato, M. 2005c. Why Are (Most) Laws of Nature Mathematical? In: J. Faye; P. Needham; U. Scheffler; M. Urchs (eds.) Nature's Principles, pp.55-75. Dordrecht: Springer.

Earman, J.; Roberts, J. 2005a. Contact with the Nomic: A Challenge for Deniers of Humean Supervenience about Laws of Nature. Part I: Humean Supervenience. Philosophy and Phenomenological Research 71(1): 1-22.

Earman, J.; Roberts, J. 2005b. Contact with the Nomic: A Challenge for Deniers of Humean Supervenience about Laws of Nature. Part II: The Epistemological Argument for Humean Supervenience. Philosophy and Phenomenological Research 71(2): 253-86.

Ellis, B. 2001. Scientific Essentialism. Oxford, Oxford University Press.

Feynman, R. 1965. The Character of a Physical Law. London, Penguin Books.

Field, H. 1980. Science without Numbers. A Defence of Nominalism. Princeton: Princeton University Press.

Field, H. 1989. Realism, Mathematics and Modality. New York: Basil Blackwell.

Giere, R. N. 1988. Explaining Science. A Cognitive Approach. Chicago and London: The University of Chicago Press. 
Giere, R. N. 1999. Science without Laws. Chicago and London: The University of Chicago Press.

Jaag, S.; Loew, C. 2018. Making Best Systems Best for Us. Synthese, DOI: https://doi.org/ 10.1007/s11229-018-1829-1.

Ladyman, J.; Ross, D.; (with D. Spurrett and J. Collier). 2007. Every Thing Must Go. Metaphysics Naturalized. Oxford: Oxford University Press.

Lange, M. 2000. Natural Laws in Scientific Practice. Oxford: Oxford University Press.

Lange, M. 2009. Laws and Lawmakers: Science, Metaphysics, and the Laws of Nature. Oxford: Oxford University Press.

Lange, M. 2017. Because without Cause. Non-Causal Explanations in Science and Mathematics. Oxford: Oxford University Press.

Lewis, D. 1986. Philosophical Papers, Volume II. New York: Oxford University Press.

Loewer, B. 1996. Humean Supervenience. Philosophical Studies 24(1): 101-27.

Massimi, M. 2018. A Perspectival Better Best System Account of Lawhood. In: W. Ott; L. Patton (eds.) Laws of Nature, pp.139-57. Oxford, Oxford University Press.

Neurath, O. 1983. Philosophical Papers 1913-1946. Vienna Circle Collection, Volume 16. New York: Kluwer, D. Reidel Publishing Company.

Nguyen, J.; Frigg, R. 2017. Mathematics is not the Only Language in the Book of Nature. Synthese, DOI: https://doi.org/10.1007/s11229-017-1526-5.

Pincock, C. 2004. A New Perspective on the Problem of Applying Mathematics. Philosophia Mathematica 3(12): 135-61

Pincock, C. 2012. Mathematics and Scientific Representation. Oxford: Oxford University Press Psillos, S. 2012. Anti-Nominalistic Scientific Realism: a Defence. In: A. Bird; B. Ellis; H. Sankey (eds.) Properties, Powers, and Structures. Issues in the Metaphysics of Realism, pp.63-80. New York and London: Routledge.

Redhead, M. 1975. Symmetry in Intertheoretical Relations. Synthese 32: 77-112.

Rizza, D. 2013. The Applicability of Mathematics: Beyond Mapping Accounts. Philosophy of Science 80(3): 398-412.

Roberts, J. 2008. The Law-Governed Universe. Oxford: Oxford University Press.

Steiner, M. 1998. The Applicability of Mathematics as a Philosophical Problem. Cambridge, MA, London, England: Harvard University Press.

Tegmark, M. 2014. Our Mathematical Universe. My Quest for the Ultimate Nature of Reality. London: Penguin Books.

van Fraassen, B. 1980. The Scientific Image. Oxford: Clarendon Press.

van Fraassen, B. 1989. Laws and Symmetry. Oxford: Clarendon Press.

Wigner, E. 1960. The Unreasonable Effectiveness of Mathematics in the Natural Sciences. Communications on Pure and Applied Mathematics 13: 1-14.

Woodward, J. 2003. Making Things Happen. A Theory of Causal Explanation. Oxford: Oxford University Press.

Woodward, J. 2013. Laws, Causes, and Invariance. In: S. Mumford; M. Tugby (eds.) Metaphysics and Science, pp.48-72. Oxford: Oxford University Press.

Woodward, J. 2017. Physical Modality, Laws, and Counterfactuals. Synthese, DOI: 10.1007/ s11229-017-1400-5.

Woodward, J. 2018. Laws: An Invariance-Based Account. In: W. Ott; L. Patton (eds.) Laws of Nature, pp.158-80. Oxford: Oxford University Press. 


\section{Notes}

${ }^{1} \mathrm{~A}$ second limit of the standard classification is that it does not naturally accommodate recent attempts at accounting for the nomological status of law statements in terms of counterfactual analysis (Lange 2000, 2009, 2017), measurements (Roberts 2008), and invariance (Woodward 2013, 2017, 2018).

${ }^{2}$ In the physics literature, Feynman (1965) provides a well-known study of the mathematical character of physical laws. He argues that most physical laws are mathematical statements providing physical information in a concise and precise manner. A reason for the contribution of mathematics to the articulation of laws is given by the plasticity with which mathematical structures express large amounts of information routinely involved in the description of complex physical situations (Dieks 2005). Feyman points out that the contribution of mathematics to the articulation of laws has come to be pervasive: "the more laws we find, and the deeper we penetrate nature, the more this disease persists. Every one of our laws is a purely mathematical statement" (Feynman 1965, p.39). Clearly, this cannot be taken literally, given that a physical law is meant to describe the physical world rather than just relations among mathematical objects. From this, however, the literature has gone farther, some contending that the formulation of physical laws is mathematical in character because reality is either ultimately mathematical (Tegmark 2014) or physical-mathematical at once (Psillos 2012). For an examination of problems for realist interpretations of mathematical structures, see Bueno (2019).

${ }^{3}$ We assume that physics is a reliable source of information about physical laws. Although philosophical reflection can undertake the task of clarifying issues about laws, it is not the role of philosophers, qua philosophers, to uncover the purportedly true laws of nature about which the laws of physics are to be deemed mere approximations. Our analysis will focus on common cases of laws of physics, such as, Hooke's law, the second dynamical law in classical mechanics, Newton's inverse-square law of universal gravitation, and the Schrödinger equation in quantum mechanics.

${ }^{4}$ See Bueno and French 2018 and Bueno and Colyvan 2011 for references and further details.

${ }^{5}$ For further details, see Bueno and French 2018, and Bueno and Colyvan 2011. In these paragraphs, we only refer to those aspects that will become central to our framework for an inferential conception of physical laws.

${ }^{6}$ This was the case in the early formulation of quantum electrodynamics, in which two mathematical formalisms were provided: one by Feynman's diagrams, as a tool for calculating particle interactions, and another by Schwinger and Tomonaga's equations for describing interacting electrons and photons. In this case, a decision was made in favor of Feynman's mathematical techniques in view of its tractability, intuitiveness, and convenience for providing understanding of the relevant structural aspects of the phenomena.

${ }^{7}$ A similar analysis can be found in Giere 1988, p.68.

${ }^{8}$ We thank Roman Frigg for pointing out this concern to us. 


\section{Acknowledgments}

We are grateful to Bruno Borge (Universidad de Buenos Aires, Argentina) and Renato Cesar Cani (Universidade Federal de Santa Catarina, Brazil) for bringing this volume together and for their continuing support. We thank audiences at the British Society for the Philosophy of Science Conference (University of Oxford, July 2018), the Workshop on foundations of Science (SADAF, Buenos Aires, Argentina, September 2018), the XI International Principia Symposium (Universidade Federal de Santa Catarina, Brazil, August 2019), and the $20^{\text {th }}$ Jornadas Rolando Chuaqui Filosofía y Ciencias (PUC de Chile, August 2019) for their stimulating feedback on earlier versions of this work. We acknowledge and thank financial support by CONICYT, Chile, through the FONDECYT 11160324 research grant. 